\title{
Significado de los valores límites en las pruebas de tolerancia a los carbohidratos en la embarazada
}

\author{
Daniel E. Londoño N., MD*; José L. Duque A., MD**
}

RESUMEN: El tamizaje para anormalidades en el metabolismo de carbohidratos es realizado frecuentemente durante el embarazo. Ciertos resultados no son estrictamente anormales.

Su manejo es controvertido. Para conocer el significado materno y fetal de estas pruebas "límite" se revisó en una cohorte histórica de 200 pacientes, las complicaciones del embarazo, parto y puerperio de dos grupos de pacientes con: 1) prueba de O'Sullivan anormal, 2) un solo valor anormal en la prueba de tolerancia oral a la glucosa y 3) se compararon entre sí y con las de un tercer grupo de pacientes con pruebas normales. También se determinó la frecuencia con que se practicaron pruebas de vigilancia materna y/o fetal para precisar sus reales ventajas y su reflejo en el resultado perinatal.

No se encontró diferencia en la frecuencia de RPM, toxemia, prematurez, macrosomía, mortinatos, infecciones de vías urinarias o amnionitis, ni de complicaciones fetales. Se observó un número mayor de estudios especiales (ecografías, doppler de cordón umbilical y monitorías fetales) en los grupos con pruebas "límite". La frecuencia de cesáreas fue similar en todos los grupos.

PAlAbRaS ClAVES: Embarazo - Prueba de tolerancia a la glucosa.

SUMMARY: Screening tests for abnormal carbohydrates metabolism during pregnancy are routinely performed. Occasionally, the results are not strictly abnormal.

In order to know the real value of this borderline results and the benefit for mother and fetus, a retrospective review of the medical records of 200 patients was performed. The patients were grouped in 1) abnormal O'Sullivan test, 2) one abnormal value in the GTT and 3) one control group with normal results. The three groups were compared for obstetrical outcome. In addition, it was determined, the use of other evaluations like routine US, doppler flow examination and electronic fetal monitoring.

There was no statistically difference in the frequency of preterm delivery, macrosomia, stillbirths, premature rupture of the membranes, toxemia, urinary tract infections or amnionitis or feta pathology.

There was a significant increase in the use of additional evaluations comparing the two groups with "border-line" tests and the control group. The frequency of cesarean section was similar in the three groups.

KEY WORDS: Pregnancy, glucose tolerance test.

Las anormalidades en el metabolismo de los carbohidratos durante el embarazo afectan a un 3\% de las pacientes. Su gran interés en la práctica obstétrica se deriva de mayor morbilidad y mortalidad cuando estas alteraciones no se detectan o no se tratan adecuadamente. Ciertas malformaciones congénitas en los hijos de madres diabéticas, la prematurez, la macrosomía, la toxemia gravídica, la pielonefritis y variadas complicaciones respiratorias, infecciosas y metabólicas en los recién nacidos llegan a ser muy graves. Sólo un diagnóstico correcto y oportuno permite un tratamiento eficaz que pueda equilibrar el resultado perinatal con el de embarazos normales.

Los criterios para el diagnóstico de diabetes gestacional son casi universales (1). En algunas pacientes las pruebas

* Residente III. Departamento de Ginecología y Obstetricia. Hospital Universitario "Lorencita Villegas de Santos". Actualmente, Especialista Hospitalario, DGORH, Fundación Santa fe de Bogotá, Colombia. Calle 116 No. 9-02. Santafé de Bogotá, D.C.

** Jefe de la Unidad de Medicina Materno-Fetal. Departamento de Ginecología y Obstetricia. Hospital Universitario "Lorencita Villegas de Santos”. Avenida 68 Carrera 40. Santafé de Bogotá, D.C. no son totalmente normales, pero tampoco cumplen los criterios para incluirlas en el diagnóstico de diabetes gestacional. Su manejo es controvertido, al punto que algunas pacientes son tratadas como diabéticas. La evidencia de que pacientes con cifras "límite" tengan una frecuencia mayor de complicaciones (maternas, fetales o neonatales) no es conclusiva. En nuestro medio no se conocen pruebas de que una valoración y manejo especiales beneficien a la madre $o$ al feto. Hacer pruebas de vigilancia materna y/o fetal en pacientes normales, asumiendo totalmente los riesgos y los costos debería reflejarse en una notable reducción de las complicaciones materno-fetales y neonatales. Tallarigo (2) observó aumento en la incidencia de macrosomía y preeclampsia en mujeres con glicemias plasmáticas más altas. Leikin (3) realizó un estudio similar, aunque solo mostró una mayor incidencia de macrosomía. Cabe mencionar el estudio de Langer (4) donde hubo una frecuencia mayor de macrosomía y problemas metabólicos neonatales, sin encontrar más diferencias entre las poblaciones estudiadas.

En esta revisión retrospectıva de 200 pacientes embarazadas se describe la frecuencia de complicaciones maternas, fetales y neonatales en un grupo de pacientes con cifras 
"límite" en las pruebas para valorar el metabolismo de carbohidratos. Así mismo se intenta establecer la utilidad de pruebas diagnósticas especiales y de vigilancia maternofetal adicionales en estos casos.

\section{Material y métodos}

Fueron revisadas 200 historias clínicas de 3 grupos de pacientes atendidas en la Unidad de Medicina Materno-Fetal del Departamento de Ginecología y Obstetricia del Hospital Infantil Universitario "Lorencita Villegas de Santos" durante el lapso comprendido entre enero de 1989 y enero de 1991. Los grupos fueron distribuidos así:

Grupo 1: Cincuenta pacientes con prueba de tamizaje (Test de O'Sullivan) anormal: superior a $140 \mathrm{mg} / \mathrm{dL}$.

Grupo 2: Cincuenta pacientes con un valor alterado en la prueba de tolerancia a la glucosa oral.

Grupo 3: Cien pacientes con pruebas de tolerancia a los carbohidratos normales.

Se excluyeron del estudio las historias clínicas de las pacientes con diabetes mellitus pregestacional, diabetes gestacional en el presente embarazo, diabetes secundaria (trauma, cirugía, etc.) o embarazo gemelar. El control del embarazo, parto, puerperio y de sus complicaciones se realizó en el hospital.

Fueron revisadas las siguientes variables: duración de la gestación en semanas, valores de la prueba de O'Sullivan y de la PTOG, número de pruebas especiales (ecografías obstétricas, monitorías fetales anteparto o intraparto, ultrasonografía doppler de cordón umbilical y amniocentesis para madurez pulmonar fetal); tipo del parto, peso del recién nacido, puntajes de Apgar, complicaciones del embarazo (amenaza de parto pretérmino, parto pretérmino, infección urinaria, pielonefritis aguda, toxemia gravídica, ruptura prematura de membranas, polihidramnios, corioamnionitis, macrosomía fetal y endometritis postparto), complicaciones del recién nacido (síndrome de dificultad respiratoria neonatal, hipoglicemia, hiperbilirrubinemia, policitemia, hipocalcemia e hipomagnesemia).

\section{Análisis estadístico}

Para comparar los promedios de los grupos se empleó la prueba Z. Para las proporciones se utilizó prueba Chi cuadrado y exacta de Fisher. Se consideró el nivel de significancia, $\mathrm{p}<0.05$.

\section{Resultados}

Las características del peso fetal en los tres grupos de pacientes, aparecen consignados en la Tabla No. 1, de esta forma:

Tabla No. 1

\begin{tabular}{|lll|}
\hline Grupo & Media & Rango \\
\hline 1 & 3107.8 & $2667-3548$ \\
2 & 3144.5 & $2695-3594$ \\
3 & 3191.9 & $2668-3514$ \\
\hline
\end{tabular}

La diferencia de peso entre los tres grupos de estudio no fue estadísticamente significativa. ( $\mathrm{p}>0.05$ ).

Ciertas complicaciones del embarazo, se presentaron en la forma que aparece en la Tabla No. 2:

Tabla No. 2

\begin{tabular}{|llll|}
\hline Grupos & $\mathbf{1}$ & $\mathbf{2}$ & $\mathbf{3}$ \\
\hline A.P.P. & $2 / 50$ & - & $3 / 100 /$ \\
Premat. & - & $2 / 50$ & $5 / 100$ \\
R.P.M. & $3 / 50$ & $3 / 50$ & $7 / 100$ \\
Toxemia & $2 / 50$ & $4 / 50$ & $6 / 100$ \\
C/Amnion. & - & - & $1 / 100$ \\
\hline
\end{tabular}

A.P.P.: Amenaza de parto pretérmino.

Premat.: Nacimientos prematuros.

C/Amnion.: Corioamnionitis.

Las patologías que más frecuentemente se asocian a un peor resultado perinatal son la prematurez y las infecciones maternas sistémicas. En este estudio no se encontró diferencia estadísticamente significativa en cuanto a prematurez. Los 2 casos de parto pretérmino en el grupo 2 corresponden a embarazos de 34.5 y 35 semanas cuyos productos desarrollaron cuadros leves de dificultad respiratoria sin presentar enfermedad de membrana hialina y evolucionaron en forma satisfactoria.

No se encontró en la revisión de las historias, casos de infección de vías urinarias ni pielonefritis y hubo un caso de corioamnionitis en el grupo de control.

No se encontró ningún caso de Apgar bajo en los grupos. No hubo mortinatos en los grupos 1 y 2 y hubo 2 casos de mortinatos en el grupo de control. La frecuencia de cesáreas en los grupos 1,2 y 3 fue de 26,30 y $24 \%$, respectivamente, sin que exista diferencia estadísticamente significativa.

El número de estudios realizado en los distintos grupos aparece en la Tabla No. 3.

Tabla No. 3

\begin{tabular}{|lrrr|}
\hline Grupos & 1 & 2 & 3 \\
\hline Ecografías & 124 & 94 & 113 \\
Doppler & 46 & 40 & 36 \\
Monitoría NST & 86 & 96 & 36 \\
Monitoría PTC & 13 & 15 & 10 \\
Amniocentesis & 2 & 1 & 1 \\
\hline
\end{tabular}

NST: Monitoría sin stress.

PTC: Prueba de tolerancia a las contracciones.

El número de estudios realizados para cada paciente se describe en la Tabla No. 4.

Tabla No. 4

\begin{tabular}{|llll|}
\hline Grupos & $\mathbf{1}$ & $\mathbf{2}$ & $\mathbf{3}$ \\
\hline Ecografías & 2.48 & 1.88 & 1.13 \\
Doppler & 0.92 & 1.12 & 0.36 \\
Monitorías & 1.66 & 1.92 & 0.36 \\
\hline
\end{tabular}

Comparando los grupos 1 y 2 para alguno de los estudios realizados, no se observa diferencia significativa. Sin embar- 
go, la comparación de cualquiera de los dos grupos anteriores con el tercero empleado como control, muestra que definitivamente sí existe diferencia, que refleja una posible tendencia a ordenar más estudios especiales que en el tercer grupo. Por ejemplo, en el grupo 1 se ordenaron dos veces más ecografías por paciente que en el 3 , y casi 4 veces más estudios Doppler, también por cada paciente. En cuanto a monitorías fetales, indispensables en la vigilancia del embarazo de alto riesgo, la tendencia es similar. Las monitorías sin stress fueron solicitadas a su vez 4 y 5 veces más frecuentemente por cada paciente en los grupos 1 y 2 , que en el 3 considerado como control

No hubo diferencia especial en el número de amniocentesis. No se detectaron malformaciones congénitas evidentes en el examen de los recién nacidos.

Tampoco hubo evidencia de complicaciones infecciosas, respiratorias o metabólicas en el período neonatal, probablemente como consecuencia de la baja tasa de partos pretérmino, que es en forma aislada, el principal responsable de estas complicaciones, independiente de la causa que lleve a prematurez.

\section{Comentarios}

Es ampliamente conocido el mal pronóstico del embarazo complicado por diabetes gestacional, ya que muy serias alteraciones en la salud materna, fetal y neonatal conllevan a altas tasas de morbimortalidad.

La única forma de evitar estos desenlaces adversos, consiste en identificar la paciente en forma oportuna, para alcanzar un control estricto de su desarreglo metabólico que permita reducir todas las patologías y complicaciones y las equilibre con la población de bajo riesgo materno-fetal.

Para asegurar el diagnóstico se debe realizar en forma rutinaria el estudio de tamizaje aceptado ampliamente, y seguir las pautas sugeridas por distintos autores (5). Las cifras propuestas se basan en estudios extensos. No hay dudas acerca del efecto favorable para la madre y para el feto de un tratamiento especial cuando se diagnostica diabetes gestacional.

Otras formas de intolerancia a los carbohidratos durante el embarazo, no han sido estudiadas tan ampliamente. Tampoco conocemos en nuestro medio estudios en embarazos con cifras "límite" en las pruebas de tolerancia a los carbohidratos, ni los resultados finales de esos embarazos.

Este estudio intenta conocer algunos aspectos del comportamiento de estos embarazos y apreciar la forma en que pruebas especiales benefician a la madre o al feto. Al hacer la revisión de los casos, es posible sugerir que ciertas entidades que comúnmente se asocian a diabetes gestacional y empeoran el pronóstico del embarazo, no son tan frecuentes cuando las pruebas de tolerancia a los carbohidratos muestran cifras "límite". Por el contrario son más bien semejantes a las que se observan en pacientes con pruebas completamente normales.

Por otra parte, observamos que la tendencia a calificar o rotular a estas pacientes como anormales, puede influir en el enfoque clínico y paraclínico por parte del equipo médico. En este estudio se muestra que estudios de vigilancia materno-fetal, empleados frecuentemente en pacientes con diabetes gestacional, fueron utilizados con una frecuencia mucho mayor que en la población general de embarazadas. Esta conducta aparentemente desprovista de mayor riesgo para la madre y para el feto, debe reconsiderarse.

En primer lugar, no debe calificarse anormal o enferma a la paciente que estando completamente asintomática, muestra como única anormalidad un examen de laboratorio "alterado". Debe buscarse en la historia clínica y en examen físico, otros datos que den cierto valor a un dato aislado del laboratorio. Si tenemos en cuenta que este grupo de pacientes con cifras "límite" no son anormales, ni tienen una frecuencia mayor de complicaciones, tampoco existiría la justificación para realizar un mayor número de estudios especiales, pues se trata de embarazos estrictamente normales. La norma de un cuidado prenatal tan especializado en pacientes con una prueba de laboratorio anormal, debiera replantearse, pues aunque los riesgos de estos estudios son realmente mínimos, los costos son asumidos totalmente por las pacientes, sin que exista evidencia clara, al menos en nuestro medio, de que esa conducta sea benéfica para la madre o para el feto.

\section{BIBLIOGRAFIA}

1. O'Sullivan JB., Mahan CM., Charles D. et al. Screening criteria for high risk gestational diabetes patients. Obstet. Gynecol. 1985; 116: 1973-1974.

2. Tallarigo L., Giampietro O., Penno G. et al. Relation of glucose tolerance to complications of pregnancy in non-diabetic women. N. Engl. J. Med. 1986; 315: 989-992.

3. Leikin EL., Jenkins JH., Pomerantz Ga. et al. Abnormal glucose screening tests in pregnancy: a risk factor for fetal macrosomia. Obstet. Gynecol. 1987; 69: 570-573.
4. Langer O., Brustman L., Anyaeg bunam A. et al. The significance of one abnormal glucose tolerance test value on adverse outcome in pregnancy. Am. J. Obstet. Gynecol. 1987; 57: 758-763.

5. Summary and recommendations of the Second International Workshop: Conference on Gestational Diabetes Mellitus, Diabetes 1985; 34(suppl 2): 123. 